\title{
VARIATION OF THE HOLOMORPHIC DETERMINANT BUNDLE
}

\author{
Julien Grivaux
}

\begin{abstract}
In this paper, we prove that the Grothendieck-Riemann-Roch formula in Deligne cohomology computing the determinant of the cohomology of a holomorphic vector bundle on the fibers of a proper submersion between abstract complex manifolds is invariant by deformation of the bundle.
\end{abstract}

\section{Introduction}

The Grothendieck-Riemann-Roch theorem is one of the cornerstones of modern algebraic geometry, and can be stated in its initial form as follows:

Theorem 1.1. [8] For any smooth quasi-projective variety $X$ over a field of characteristic zero, the morphism $\mathcal{F} \rightarrow \operatorname{ch}(\mathcal{F}) \operatorname{Td}(X)$ from the Grothendieck group $\mathrm{K}(X)$ of coherent sheaves on $X$ to the Chow ring $\mathrm{CH}(X)$ of $X$ commutes with proper pushforward.

Since Serre's $[28,29]$ fundamental papers on coherent sheaves, it has become natural and useful to translate results from algebraic to analytic geometry. Concerning the Grothendieck-Riemann-Roch theorem, this has been the object of many researches from early 60 s till the 80 s, starting with the case of analytic immersions [2] and pursuing with the index theorem for vector bundles and coherent analytic sheaves (see $[3,23,25,33])$. The outcome of these works is O'Brian-Toledo-Tong's proof of the Grothendieck-Riemann-Roch theorem in Hodge cohomology for arbitrary proper holomorphic maps between complex manifolds [24]. By completely different methods, Levy [22] succeeded in proving the analogous statement in De Rham cohomology, where the Chern classes are constructed by means of locally free resolutions in the category of real-analytic coherent sheaves.

From the middle of the 1980s, some new ideas about the Grothendieck-RiemannRoch theorem emerged after the seminal article of Quillen [26] introducing canonical hermitian metrics on determinant bundles associated with the cohomology of a vector bundle on the fibers of a holomorphic submersion (see [30, Chapter VI]). Building on initial results in the case of families of curves (see [5,9,26]), Bismut, Gillet and Soulé [6] proved that, for locally Kähler fibrations, the curvature of this determinant bundle is exactly given by the component of degree two of the Grothendieck-Riemann-Roch theorem at the level of differential forms. This theorem has been extended to all degrees in [7] provided that the higher direct images of the bundle are locally free. Quite recently, Bismut succeeded in removing the kählerianity assumption on the morphism and obtained the following result:

Received by the editors February 13, 2013. 
Theorem 1.2. [4, Theorem 0.1.1] Let $f: X \rightarrow Y$ be a proper holomorphic submersion between complex manifolds and $\mathcal{E}$ be a holomorphic vector bundle on $X$. Then the following identities hold in the Bott-Chern cohomology ring of $Y$ :

(i) $\mathrm{c}_{1}^{\mathrm{BC}}\left[\operatorname{det} \mathrm{R} f_{*} \mathcal{E}\right]=\left(f_{*}\left\{\operatorname{ch}^{\mathrm{BC}}(\mathcal{E}) \operatorname{td}^{\mathrm{BC}}\left(\mathrm{T}_{X / Y}\right)\right\}\right)^{(1,1)}$ in $\mathrm{H}_{\mathrm{BC}}^{1,1}(Y)$.

(ii) If all the sheaves $\mathrm{R}^{i} f_{*} \mathcal{E}$ are locally free, then

$$
\operatorname{ch}^{\mathrm{BC}}\left(\mathrm{R} f_{*} \mathcal{E}\right)=f_{*}\left\{\operatorname{ch}^{\mathrm{BC}}(\mathcal{E}) \operatorname{td}^{\mathrm{BC}}\left(\mathrm{T}_{X / Y}\right)\right\} \text { in } \oplus_{p \geq 0} \mathrm{H}_{\mathrm{BC}}^{p, p}(Y) .
$$

On abstract complex manifolds, one of the finest known cohomology theory where Chern classes exist for holomorphic vector bundles is Deligne-Beulinson cohomology. The ultimate goal of our program would be to prove the Grothendieck-Riemann-Roch theorem in this cohomology. The statement does not immediately make sense even for holomorphic vector bundles, because Chern classes must be defined for the direct images sheaves $\mathrm{R}^{i} f_{*} \mathcal{E}$. The problem of defining Chern classes of coherent sheaves in Deligne cohomology is solved on compact manifolds in [14]. The corresponding Grothendieck-Riemann-Roch theorem is proved for projective morphisms between complex compact manifolds.

In this paper, we focus on the component of degree two on the base of the Grothendieck-Riemann-Roch theorem in Deligne cohomology for holomorphic vector bundles. Our main result describes explicitly the variation of the determinant bundle, first in the rational Deligne cohomology group $\mathrm{H}_{\mathrm{D}}^{2}(Y, \mathbb{Q}(1))$ and then in Pic $(Y)$ under mild assumptions on $Y$ :

Theorem 1.3. Let $f: X \rightarrow Y$ be a proper holomorphic submersion between complex manifolds $X, Y$, and let $\left(\mathcal{E}_{t}\right)_{t \in \Delta}$ be a holomorphic family of holomorphic vector bundles on $X$ parameterized by the complex unit disc $\Delta$.

(i) Rational variation formula

For any $s$ and $t$ in $\Delta$,

$$
\mathrm{c}_{1}^{\mathrm{D}}\left[\operatorname{det} \mathrm{R} f_{*} \mathcal{E}_{s}\right]-\mathrm{c}_{1}^{\mathrm{D}}\left[\operatorname{det} \mathrm{R} f_{*} \mathcal{E}_{t}\right]=\left(f_{*}\left\{\left[\operatorname{ch}^{\mathrm{D}}\left(\mathcal{E}_{s}\right)-\operatorname{ch}^{\mathrm{D}}\left(\mathcal{E}_{t}\right)\right] \operatorname{td}^{\mathrm{D}}\left(\mathrm{T}_{X / Y}\right)\right\}\right)^{(2)}
$$

in the rational Deligne cohomology group $\mathrm{H}_{\mathrm{D}}^{2}(Y, \mathbb{Q}(1))$.

(ii) Integral variation formula

If $\mathrm{H}^{1}\left(Y, \mathcal{O}_{Y}\right)$ is separated and if $\mathrm{H}^{1}\left(Y, \mathbb{Q}_{Y}\right)$ is countable, then there exists a unique analytic curve $\gamma$ from $\Delta$ to $\mathrm{Pic}^{0}(Y)$ vanishing at 0 such that for any $t$ in $\Delta$,

$$
\mathrm{c}_{1}^{\mathrm{D}}(\gamma(t))=\left(f_{*}\left\{\left[\operatorname{ch}^{\mathrm{D}}\left(\mathcal{E}_{t}\right)-\operatorname{ch}^{\mathrm{D}}\left(\mathcal{E}_{0}\right)\right] \operatorname{td}^{\mathrm{D}}\left(\mathrm{T}_{X / Y}\right)\right\}\right)^{(2)}
$$

in $\mathrm{H}_{\mathrm{D}}^{2}(Y, \mathbb{Q}(1))$. For any $s$ and $t$ in $\Delta$,

$$
\left[\operatorname{det} \mathrm{R} f_{*} \mathcal{E}_{s}\right]-\left[\operatorname{det} \mathrm{R} f_{*} \mathcal{E}_{t}\right]=\gamma(s)-\gamma(t) .
$$

Remark that since $\mathrm{H}^{1}(Y, \mathbb{Q})$ is isomorphic to $\operatorname{Hom}_{\mathbb{Z}}\left(\mathrm{H}_{1}(Y, \mathbb{Z}), \mathbb{Q}\right)$, it is sufficient to require that the torsion-free part of $\mathrm{H}_{1}(Y, \mathbb{Z})$ is finitely generated to ensure that $\mathrm{H}^{1}(Y, \mathbb{Q})$ is countable. This condition can be easier to check in concrete situations.

Even if $\operatorname{Pic}(Y)$ is not always a complex manifold, it is possible to give a precise definition of an analytic curve with values in $\operatorname{Pic}(Y)$ that matches with the usual one when the image of $\mathrm{H}^{1}(Y, \mathbb{Z})$ is discrete in $\mathrm{H}^{1}\left(Y, \mathcal{O}_{Y}\right)$. 
On the one hand, Theorem 1.3 is motivated by Teleman's program on the classification of class VII surfaces (see [31,32]). On the other hand, it is a little step toward the general Grothendieck-Rieman-Roch theorem in Deligne cohomology (which is currently out of reach in full generality). For instance, we have the following result:

Theorem 1.4. Let $Y$ and $F$ be complex manifolds such that $F$ is compact of complex dimension $n$, and let $p: Y \times F \rightarrow Y$ and $q: Y \times F \rightarrow F$ be the two natural projections. Then, for any $\mathcal{L}$ in $\operatorname{Pic}^{0}(Y \times F)$,

$$
\mathrm{c}_{1}^{\mathrm{D}}\left[\operatorname{det} \mathrm{R} p_{*} \mathcal{L}\right]=p_{*}\left\{\mathrm{c}_{1}^{\mathrm{D}}(\mathcal{L}) q^{*} \operatorname{td}_{n}^{\mathrm{D}}(F)\right\}
$$

in the rational Deligne cohomology group $\mathrm{H}_{\mathrm{D}}^{2}(Y, \mathbb{Q}(1))$.

The paper is organized as follows: in Section 2 we provide a short recollection of operations on derived categories in the analytic setting. Then we recall the theory of determinants for coherent analytic sheaves (see [19], [20, Chapter 5, Section 6] and [6, Section 3]) and we prove in Proposition 2.4 a base change formula for determinant bundles. We also prove a folklore result (Lemma 2.2) saying that the first Chern class of a coherent sheaf in Hodge cohomology is the same as the first Chern class of its determinant. In Section 3, we recall the basics of Deligne cohomology (see [11] and [34, Chapter 12]). Proposition 3.1 is the main ingredient of the proof of Theorem 1.3. Then we discuss analytic curves with values in the Picard group of a complex manifold. Lastly, Section 4 is devoted to the proofs of Theorems 1.3 and 1.4.

\section{Derived categories and determinant bundles}

2.1. Derived categories in the analytic setting. For generalities on sheaves and derived categories, we refer the reader to $[10,12,17]$. For any connected complex manifold $X$, let $\mathrm{D}^{+}(X)$ (resp. $\mathrm{D}^{-}(X)$, resp. $\mathrm{D}^{\mathrm{b}}(X)$ ) denote the right-bounded (resp. left-bounded, resp. bounded) derived category of analytic sheaves on $X$; and let $\mathrm{D}_{\mathrm{coh}}^{\mathrm{b}}(X)$ be the full subcategory of $\mathrm{D}^{\mathrm{b}}(X)$ consisting of bounded complexes with coherent cohomology. Recall that the category of sheaves over any sheaf of rings has enough injective and flat objects [17, Propositions 2.4.3 and 2.4.12]. Let $f: X \rightarrow Y$ be a holomorphic map. Then there exists a derived pullback morphism

$$
\mathrm{L} f^{*}: \mathrm{D}^{-}(Y) \rightarrow \mathrm{D}^{-}(X)
$$

given by $\mathrm{L} f^{*} \mathcal{F}=f^{-1} \mathcal{F} \stackrel{\mathrm{L}}{\otimes}_{f-1} \mathcal{O}_{Y} \mathcal{O}_{X}$, where $\stackrel{\mathrm{L}}{\otimes}$ denotes the derived tensor product. If $f$ is flat, we write $f^{*}$ instead of $\mathrm{L} f^{*}$. Since $\mathcal{O}_{X}$ is quasi-isomorphic to a bounded complex of flat $f^{-1} \mathcal{O}_{Y}$-modules, $\mathrm{L} f^{*}$ maps $\mathrm{D}^{\mathrm{b}}(Y)$ to $\mathrm{D}^{\mathrm{b}}(X)$. Besides, $\mathrm{L} f^{*}$ maps $\mathrm{D}_{\text {coh }}^{\mathrm{b}}(Y)$ to $\mathrm{D}_{\text {coh }}^{\mathrm{b}}(X)$. On the other hand, there is a derived push-forward morphism

$$
\mathrm{R} f_{*}: \mathrm{D}^{+}(X) \rightarrow \mathrm{D}^{+}(Y) .
$$

If $f$ is a proper holomorphic map, thanks to Grauert's [13] finiteness theorem, $\mathrm{R} f_{*}$ maps $\mathrm{D}_{\text {coh }}^{\mathrm{b}}(X)$ to $\mathrm{D}_{\text {coh }}^{\mathrm{b}}(Y)$. The derived pullback and push-forward functors are related via the projection formula: if $\mathcal{F}$ and $\mathcal{G}$ are elements of $\mathrm{D}_{\text {coh }}^{\mathrm{b}}(Y)$ and $\mathrm{D}_{\text {coh }}^{\mathrm{b}}(X)$ respectively and if $f$ is proper, there is a canonical isomorphism in $\mathrm{D}_{\text {coh }}^{\mathrm{b}}(Y)$ :

$$
\mathrm{R} f_{*} \mathcal{G} \stackrel{\mathrm{L}}{\otimes}_{\mathcal{O}_{Y}} \mathcal{F} \simeq \mathrm{R} f_{*}\left(\mathcal{G} \stackrel{\mathrm{L}}{\otimes}_{\mathcal{O}_{X}} \mathrm{~L} f^{*} \mathcal{F}\right) .
$$


This isomorphism is called the projection formula. We refer the reader to the proof of [17, Proposition 2.6.6] which is valid in this context.

2.2. Determinants for coherent sheaves. Let $\mathcal{F}$ be a coherent analytic sheaf on a connected complex manifold $X$. The determinant of $\mathcal{F}$, denoted by $\operatorname{det} \mathcal{F}$, is a holomorphic line bundle on $X$ defined as follows:

- If $\mathcal{F}$ is torsion-free, there exists a Zariski-open subset $U$ of $X$ such that $\mathcal{F}$ is locally free on $U$ and $X \backslash U$ has codimension at least two in $X$. Then the top exterior power of $\mathcal{F}_{\mid U}$ is a holomorphic line bundle on $U$, which extends uniquely to a line bundle $\operatorname{det} \mathcal{F}$ on $X$.

- If $\mathcal{F}$ is a torsion sheaf, let $Z$ be the maximal closed hypersurface contained in $\operatorname{supp} \mathcal{F}$, let $\left(Z_{i}\right)_{i \in I}$ be the irreducible components of $Z$, and let $\mathfrak{I}_{i}$ be the ideal sheaf defining $Z_{i}$. For any integer $k$, the sheaf $\mathfrak{I}_{i}^{k} \mathcal{F} / \mathfrak{I}_{i}^{k+1} \mathcal{F}$ is the pushforward of a coherent sheaf on $Z_{i}$, we denote its generic rank by $r_{i}(k)$. If $m_{i}=\sum_{k} r_{i}(k)$, then the determinant of $\mathcal{F}$ is given by the formula

$$
\operatorname{det} \mathcal{F}=\bigotimes_{i} \mathcal{O}_{X}\left(m_{i} Z_{i}\right)
$$

- If $\mathcal{F}$ is arbitrary and $\mathcal{F}_{\text {tors }}$ is its maximal torsion subsheaf, then $\mathcal{F} / \mathcal{F}_{\text {tors }}$ is torsion-free and

$$
\operatorname{det} \mathcal{F}=\operatorname{det}\left(\mathcal{F} / \mathcal{F}_{\text {tors }}\right) \otimes \operatorname{det} \mathcal{F}_{\text {tors }}
$$

Recall that it is also possible to construct determinants of coherent sheaves using local free resolutions. We refer the reader to [20, Chapter 5 Section 6] for this approach. The main property of determinants is the following [20, Chapter 5 Proposition 6.9]: for any bounded complex $\mathcal{K}^{\bullet}$ of coherent sheaves on $X$, using additive notation for line bundles, we have a canonical isomorphism

$$
\sum_{i}(-1)^{i} \operatorname{det} \mathcal{K}^{i} \simeq \sum_{i}(-1)^{i} \operatorname{det} \mathcal{H}^{i}\left(\mathcal{K}^{\bullet}\right)
$$

For any bounded complex $\mathcal{K}^{\bullet}$ on $X$ with coherent cohomology, the determinant of $\mathcal{K}^{\bullet}$ is defined by the formula $\operatorname{det} \mathcal{K}^{\bullet}=\sum_{i}(-1)^{i} \operatorname{det} \mathcal{H}^{i}\left(\mathcal{K}^{\bullet}\right)$. Two quasi-isomorphic bounded complexes with coherent cohomology have canonically isomorphic determinants. Therefore, the determinant of an object in $\mathrm{D}_{\mathrm{coh}}^{\mathrm{b}}(X)$ is well-defined, and two isomorphic objects have the same determinant. Besides, the determinant map factorizes through the Grothendieck group $\mathrm{K}(X)$ of the category of coherent sheaves on $X$.

Lemma 2.1. Let $\varphi: X \rightarrow Y$ be a holomorphic map between connected complex manifolds. Then for any element $\mathcal{K}$ in $\mathrm{D}_{\mathrm{coh}}^{\mathrm{b}}(Y), \varphi^{*} \operatorname{det} \mathcal{K} \simeq \operatorname{det}\left(\mathrm{L} \varphi^{*} \mathcal{K}\right)$.

Proof. It is enough to prove the lemma when $\mathcal{K}$ is a single coherent sheaf in degree zero. For any Stein subset $U$ of $Y$, let $\mathcal{E}^{\bullet}$ be a locally free resolution of $\mathcal{K}_{\mid U}$. Then we have canonical isomorphisms

$$
\varphi^{*} \operatorname{det} \mathcal{K}_{\mid U} \simeq \varphi^{*}\left[\sum_{i}(-1)^{i} \operatorname{det}\left(\mathcal{E}^{i}\right)\right]=\sum_{i}(-1)^{i} \operatorname{det}\left(\varphi^{*} \mathcal{E}^{i}\right)=\operatorname{det}\left(\mathrm{L} \varphi^{*} \mathcal{K}_{\mid U}\right)
$$

which can be glued together by a standart procedure (see, e.g., [20, pp. 163-165]) to give a global isomorphism on $X$ between $\varphi^{*} \operatorname{det} \mathcal{K}$ and $\operatorname{det}\left(\mathrm{L} \varphi^{*} \mathcal{K}\right)$. 
For any coherent sheaf $\mathcal{F}$ on $X$, we denote by $\mathrm{c}_{i}^{\mathrm{H}}(\mathcal{F})$ the Chern classes of $\mathcal{F}$ in $\mathrm{H}^{i}\left(X, \Omega_{X}^{i}\right)$ and by $\operatorname{ch}^{\mathrm{H}}(\mathcal{F})$ its Chern character in the total Hodge cohomology ring of $X$.

Lemma 2.2. For any complex manifold $X$ and any coherent analytic sheaf $\mathcal{F}$ on $X$, we have $\mathrm{c}_{1}^{\mathrm{H}}(\mathcal{F})=\mathrm{c}_{1}^{\mathrm{H}}(\operatorname{det} \mathcal{F})$ in $\mathrm{H}^{1}\left(X, \Omega_{X}^{1}\right)$.

Proof. We can assume without loss of generality that $\mathcal{F}$ is either a torsion sheaf or a torsion-free sheaf. Thanks to the vanishing theorem for local cohomology $[8$, Theorem 3.6], for any analytic set $W$ of codimension at least two in $X$, the natural map from $\mathrm{H}^{1}\left(X, \Omega_{X}^{1}\right)$ to $\mathrm{H}^{1}\left(X \backslash W, \Omega_{X \backslash W}^{1}\right)$ is injective. Thus, if $U$ is a Zariski-open subset of $X$ such that $\operatorname{codim}_{X}(X \backslash U) \geq 2$, it is enough to prove that $\mathrm{c}_{1}^{\mathrm{H}}\left(\mathcal{F}_{\mid U}\right)=\mathrm{c}_{1}^{\mathrm{H}}\left(\operatorname{det} \mathcal{F}_{\mid U}\right)$. Now we have to deal with two different cases:

- First case: the sheaf $\mathcal{F}$ is a torsion sheaf whose support is a smooth hypersurface. Since $\mathcal{F}=\sum_{k} \mathfrak{I}_{Z}^{k} \mathcal{F} / \mathfrak{I}_{Z}^{k+1} \mathcal{F}$ in $\mathrm{K}(X)$, we can assume without loss of generality that $\mathfrak{I}_{Z} \mathcal{F}=0$. After removing again an analytic subset of $X$ of codimension at least two, we can even assume that $\mathcal{F}$ is the push-forward of a locally free sheaf of rank $r$ on $Z$. This implies $\operatorname{det} \mathcal{F}=\mathcal{O}_{X}(r Z)$. Then, using the Grothendieck-Riemann-Roch theorem in Hodge cohomology for immersions [24], we get $\mathrm{c}_{1}^{\mathrm{H}}(\mathcal{F})=r[Z]_{\mathrm{H}}=\mathrm{c}_{1}^{\mathrm{H}}\left(\mathcal{O}_{X}(r Z)\right)=\mathrm{c}_{1}^{\mathrm{H}}(\operatorname{det} \mathcal{F})$.

- Second case: the sheaf $\mathcal{F}$ is locally free. Then it is well-know that $\mathrm{c}_{1}^{\mathrm{H}}(\mathcal{F})=$ $c_{1}^{\mathrm{H}}(\operatorname{det} \mathcal{F})$.

2.3. Base change. Let $f: X \rightarrow Y$ be a proper holomorphic submersion between two connected complex manifolds $X$ and $Y$, and let $\mathcal{E}$ be a locally free sheaf on $X$.

Definition 2.3. The determinant of the cohomology ${ }^{1} \lambda(\mathcal{E}, f)$ attached to the couple $(\mathcal{E}, f)$ is the class of $\operatorname{det}\left(\operatorname{R} f_{*} \mathcal{E}\right)$ in $\operatorname{Pic}(Y)$.

We now state and prove a base change theorem for the determinant of the cohomology. Let $T$ be a complex manifold and $u: T \rightarrow Y$ be a closed immersion of complex manifolds, and consider the cartesian diagram

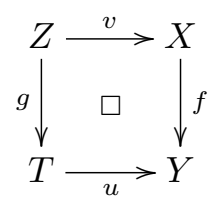

Proposition 2.4. For any vector bundle $\mathcal{E}$ on $X, u^{*} \lambda(\mathcal{E}, f)=\lambda\left(v^{*} \mathcal{E}, g\right)$ in $\operatorname{Pic}(T)$.

Proof. Since $f$ and $g$ are holomorphic submersions, they are flat morphisms. Using the projection formula (2.1) twice, we have in $\mathrm{D}_{\text {coh }}^{\mathrm{b}}(Y)$

$$
\begin{aligned}
u_{*}\left(\mathrm{~L} u^{*} \mathrm{R} f_{*} \mathcal{E}\right) & \simeq \mathrm{R} f_{*} \mathcal{E} \stackrel{\mathrm{L}}{\otimes}_{\mathcal{O}_{Y}} u_{*} \mathcal{O}_{T} \simeq \mathrm{R} f_{*}\left(\mathcal{E} \stackrel{\mathrm{L}}{\otimes}_{\mathcal{O}_{X}} f^{*} u_{*} \mathcal{O}_{T}\right) \simeq \mathrm{R} f_{*}\left(\mathcal{E} \stackrel{\mathrm{L}}{\otimes}_{\mathcal{O}_{X}} v_{*} g^{*} \mathcal{O}_{T}\right) \\
& \simeq \mathrm{R} f_{*} \mathrm{R} v_{*}\left(v^{*} \mathcal{E} \stackrel{\mathrm{L}}{\otimes}_{\mathcal{O}_{Z}} g^{*} \mathcal{O}_{T}\right) \simeq \mathrm{R} u_{*} \mathrm{R} g_{*}\left(v^{*} \mathcal{E}{\stackrel{\mathrm{L}}{\mathcal{O}_{Z}}} g^{*} \mathcal{O}_{T}\right) \\
& \simeq u_{*}\left[\mathrm{R} g_{*}\left(v^{*} \mathcal{E}\right)\right] .
\end{aligned}
$$

\footnotetext{
${ }^{1}$ It is also called Knudsen-Mumford determinant in the literature.
} 
This proves that for any integer $i$,

$$
\mathcal{H}^{i}\left(\mathrm{~L} u^{*} \mathrm{R} f_{*} \mathcal{E}\right) \simeq \mathrm{R}^{i} g_{*}\left(v^{*} \mathcal{E}\right) .
$$

Then we can conclude using Lemma 2.1.

\section{Deligne cohomology and Picard group}

3.1. Deligne classes of degree 2. For any complex manifold X and any nonnegative integer $p$, the Deligne complex $\mathbb{Z}_{\mathrm{D}, X}(p)$ is the complex

$$
\mathbb{Z}_{X} \stackrel{(2 \mathbf{i} \pi)^{p}}{\longrightarrow} \mathcal{O}_{X} \stackrel{\partial}{\longrightarrow} \Omega_{X}^{1} \stackrel{\partial}{\longrightarrow} \cdots \stackrel{\partial}{\longrightarrow} \Omega_{X}^{p-1}
$$

where the constant sheaf $\mathbb{Z}_{X}$ sits in degree zero. The integral Deligne cohomology groups of $X$ are defined by the formula $\mathrm{H}_{\mathrm{D}}^{k}(X, \mathbb{Z}(p))=\mathbb{H}^{k}\left(X, \mathbb{Z}_{\mathrm{D}, \mathrm{X}}(p)\right)$. Similar definitions hold for the rational Deligne complex $\mathbb{Q}_{\mathrm{D}, X}(p)$ as well as for the rational Deligne cohomology groups $\mathrm{H}_{\mathrm{D}}^{k}(X, \mathbb{Q}(p))$. For any locally free sheaf $\mathcal{E}$ on $X$, we will denote by $\mathrm{c}_{i}^{\mathrm{D}}(\mathcal{E})$ the rational Chern classes of $\mathcal{E}$ in $\mathrm{H}_{\mathrm{D}}^{2 i}(X, \mathbb{Q}(i))$, and by $\operatorname{ch}^{\mathrm{D}}(\mathcal{E})$ the Chern character of $\mathcal{E}$.

Let Pic $(X)$ be the Picard group of $X$ classifying isomorphism classes of line bundles on $X$, it is isomorphic to $\mathrm{H}^{1}\left(X, \mathcal{O}_{X}^{*}\right)$. Since $\mathbb{Z}_{D, X}(1)$ is quasi-isomorphic to $\mathcal{O}_{X}^{*}[-1]$, $\mathrm{H}_{\mathrm{D}}^{2}(X, \mathbb{Z}(1))$ is isomorphic to Pic $(X)$. Note that the kernel of

$$
\mathrm{c}_{1}^{\mathrm{D}}: \operatorname{Pic}(X) \simeq \mathrm{H}_{\mathrm{D}}^{2}(X, \mathbb{Z}(1)) \rightarrow \mathrm{H}_{\mathrm{D}}^{2}(X, \mathbb{Q}(1))
$$

is exactly the maximal torsion subgroup of $\operatorname{Pic}(X)$.

There is a natural cup-product in Deligne cohomology (cf [11]). Besides, for any nonnegative integer $p$, the morphism $\partial: \Omega_{X}^{p-1} \rightarrow \Omega_{X}^{p}$ induces a morphism from $\mathbb{Q}_{\mathrm{D}, \mathrm{X}}(p)$ to $\Omega_{X}^{p}[-p]$. Hence for any nonnegative integer $k$, we obtain a natural map from $\mathrm{H}_{\mathrm{D}}^{k+p}(X, \mathbb{Q}(p))$ to $\mathrm{H}^{k}\left(X, \Omega_{X}^{p}\right)$ which is compatible with cup-products on both sides.

Let us now give the key ingredient of the proof of Theorem 1.3.

Proposition 3.1. Let $\Delta$ be the complex unit disc, let $X$ be a complex manifold and let $\alpha$ be a class in $\mathrm{H}_{\mathrm{D}}^{2}(X \times \Delta, \mathbb{Q}(1))$. The class $\alpha$ maps to 0 via the morphism

$$
\mathrm{H}_{\mathrm{D}}^{2}(X \times \Delta, \mathbb{Q}(1)) \rightarrow \mathrm{H}^{1}\left(X \times \Delta, \Omega_{X \times \Delta}^{1}\right) \rightarrow \mathrm{H}^{1}\left(X \times \Delta, \mathcal{O}_{X} \otimes \Omega_{\Delta}^{1}\right)
$$

if and only if there exists a class $\beta$ in $\mathrm{H}_{\mathrm{D}}^{2}(X, \mathbb{Q}(1))$ such that $\alpha=\operatorname{pr}_{1}^{*} \beta$.

Proof. Let $p: X \times \Delta \rightarrow X$ be the first projection. By the projection formula,

$$
\mathrm{R} p_{*}\left\{p^{-1} \mathbb{Q}_{\mathrm{D}, X}(1)\right\} \simeq \mathbb{Q}_{\mathrm{D}, X}(1) \stackrel{\mathrm{L}}{\otimes}_{\mathbb{Q}_{X}} \mathrm{R} p_{*} \mathbb{Q}_{X \times \Delta} \simeq \mathbb{Q}_{\mathrm{D}, X}(1),
$$

so that we obtain the isomorphisms

$$
\mathrm{R} \Gamma\left(X \times \Delta, \mathrm{p}^{-1} \mathbb{Q}_{\mathrm{D}, X}(1)\right) \simeq \mathrm{R} \Gamma\left(X, \mathrm{Rp}_{*}\left\{p^{-1} \mathbb{Q}_{\mathrm{D}, X}(1)\right\}\right) \simeq \mathrm{R} \Gamma\left(X, \mathbb{Q}_{\mathrm{D}, X}(1)\right) .
$$

We have a natural exact sequence of complexes

$$
0 \longrightarrow p^{-1} \mathbb{Q}_{\mathrm{D}, X}(1) \stackrel{p^{*}}{\longrightarrow} \mathbb{Q}_{\mathrm{D}, X \times \Delta}(1) \longrightarrow \mathcal{O}_{X} \otimes \Omega_{\Delta}^{1}[-1] \longrightarrow 0,
$$

which is given by the isomorphism $p^{-1} \mathbb{Q}_{X} \stackrel{\sim}{\sim} \mathbb{Q}_{X \times \Delta}$ in degree zero and by the sequence

$$
0 \longrightarrow p^{-1} \mathcal{O}_{X} \longrightarrow \mathcal{O}_{X \times \Delta} \stackrel{d_{t}}{\longrightarrow} \mathcal{O}_{X} \otimes \Omega_{\Delta}^{1} \longrightarrow 0
$$


in degree one. Writing down the long cohomology exact sequence, we get the following short exact sequence:

$$
\mathrm{H}_{\mathrm{D}}^{2}(X, \mathbb{Q}(1)) \stackrel{p^{*}}{\longrightarrow} \mathrm{H}_{\mathrm{D}}^{2}(X \times \Delta, \mathbb{Q}(1)) \longrightarrow \mathrm{H}^{1}\left(X \times \Delta, \mathcal{O}_{X} \otimes \Omega_{\Delta}^{1}\right) .
$$

This gives the result.

\section{Remark 3.2.}

(i) Proposition 3.1 is also valid for integral Deligne cohomogy.

(ii) It possible to prove Proposition 3.1 by a purely geometric argument. We can assume without loss of generality that $\alpha$ is the first Chern class of a vector bundle $\mathcal{L}$ in $\operatorname{Pic}(X \times \Delta)$. The vanishing of the relative Atiyah class of $\mathcal{L}$ in $\mathrm{H}^{1}\left(X \times \Delta, \mathcal{O}_{X} \otimes \Omega_{\Delta}^{1}\right)$ means that there exists a relative holomorphic connection $\nabla: \mathcal{L} \rightarrow \mathcal{L} \otimes \operatorname{pr}_{2}^{*} \Omega_{\Delta}^{1}$ on $\mathcal{L}$. This relative connexion defines a true holomorphic connexion on each slice $\{x\} \times \Delta$, which is automatically flat; so that the restriction of $\mathcal{L}$ to this slice is canonically trivial. This proves that $\mathcal{L}$ is globally isomorphic to $\mathrm{p}^{*} \mathcal{L}_{\mid X \times\{0\}}$, and we take for $\beta$ the class of $\mathcal{L}_{\mid X \times\{0\}}$ in $\operatorname{Pic}(X)$.

(iii) For a geometric interpretation of (i) using analytic curves with values in the Picard group, we refer the reader to Proposition 3.5 (iii).

3.2. Analytic curves in the Picard group. In this section, we discuss the notion of analytic curve with values in the Picard group of a complex manifold. This problem must be dealt with carefully. Let us first recall that there is an exact sequence

$$
0 \longrightarrow \operatorname{Pic}^{0}(X) \longrightarrow \operatorname{Pic}(X) \stackrel{\mathrm{c}_{1}}{\longrightarrow} \mathrm{H}^{2}(X, \mathbb{Z}),
$$

where $\operatorname{Pic}^{0}(X)=\mathrm{H}^{1}\left(X, \mathcal{O}_{X}\right) / 2 \mathbf{i} \pi \mathrm{H}^{1}(X, \mathbb{Z}) \simeq \mathrm{H}^{1}\left(X, \mathcal{O}_{X}\right) / \mathrm{H}^{1}(X, \mathbb{Z})$, the last isomorphism being given by $\alpha \rightarrow \alpha / 2 \mathbf{i} \pi$. Remark that $\mathrm{H}^{1}\left(X, \mathcal{O}_{X}\right)$ carries a natural topology from its representation using Dolbeault cohomology. Let us put the discrete topology on $\mathrm{H}^{2}(X, \mathbb{Z})$. Then we can endow $\operatorname{Pic}(X)$ with a canonical topology, which is defined by requiring that all arrows in $(3.2)$ are continuous.

If $X$ is compact, the group $\mathrm{H}^{1}\left(X, \mathcal{O}_{X}\right) / \mathrm{H}^{1}\left(X, \mathbb{Z}_{X}\right)$ is a genuine complex Lie group. Indeed, if $\alpha$ is a De Rham representative of a cohomology class in $\mathrm{H}^{1}(X, \mathbb{R})$, then $\alpha^{0,1}$ is a Dolbeault representative of the image of $[\alpha]$ in $\mathrm{H}^{1}\left(X, \mathcal{O}_{X}\right)$. Therefore, if $\alpha^{0,1}=\bar{\partial} f$ where $f=u+\mathbf{i} v$ is a smooth complex-valued function on $X$, then $\alpha=\mathrm{d} u+\mathrm{d}^{\mathrm{c}} v$. It follows that $\mathrm{dd}^{\mathrm{c}} v=0$ and since $X$ is compact, $v$ is constant. Hence $\mathrm{H}^{1}(X, \mathbb{R})$ injects in $\mathrm{H}^{1}\left(X, \mathcal{O}_{X}\right)$. Since $\mathrm{H}^{1}(X, \mathbb{R})$ and $\mathrm{H}^{1}\left(X, \mathcal{O}_{X}\right)$ are finite-dimensional, the torsion-free part of $\mathrm{H}^{1}(X, \mathbb{Z})$ is a discrete subgroup of $\mathrm{H}^{1}\left(X, \mathcal{O}_{X}\right)$.

If $X$ is not compact the natural topology on Pic $(X)$ is not Hausdorff in general. This comes from the fact that the Dolbeault cohomology groups of a complex manifold are no longer separated. For instance, there is a counterexample due to Rossi [27] of a two-dimensional complex manifold $X$ such that $\mathrm{H}^{1}\left(X, \mathcal{O}_{X}\right)$ is not separated (see [21] for more details on this topic). And of course, even if $\mathrm{H}^{1}\left(X, \mathcal{O}_{X}\right)$ is separated, the image of $\mathrm{H}^{1}(X, \mathbb{Z})$ is not a priori discrete in $\mathrm{H}^{1}\left(X, \mathcal{O}_{X}\right)^{2}$.

Let $\Delta$ denote the complex unit disk. We make the following definition, which is intuitively very natural:

\footnotetext{
${ }^{2}$ The author was told by M. Verbitsky that a well-chosen nilmanifold would probably provide such an example.
} 
Definition 3.3. For any complex manifold $X$, a curve $\gamma: \Delta \rightarrow \operatorname{Pic}(X)$ is analytic if there exists an element $\alpha$ in Pic $(X \times \Delta)$ such that for all $t$ in $\Delta, \gamma(t)=\alpha_{\mid X \times\{t\}}$.

This definition is of little use if $\mathrm{H}^{1}\left(X, \mathcal{O}_{X}\right)$ is not separated, and we will avoid this pathological case. In what follows, we will use a little bit of the theory of holomorphic functions of one complex variable with values in a Fréchet space. We refer the interested reader to the papers $[15,16]$ for a complete account of this theory. If $X$ is compact, these subtleties are no longer needed.

Proposition 3.4. Let $X$ be a complex manifold, assume that $\mathrm{H}^{1}\left(X, \mathcal{O}_{X}\right)$ is separated, and let $\gamma: \Delta \rightarrow \operatorname{Pic}(X)$ be a curve.

(i) The curve $\gamma$ is analytic if and only if $\gamma-\gamma(0)$ can be lifted to an holomorphic curve with values in the Fréchet space $\mathrm{H}^{1}\left(X, \mathcal{O}_{X}\right)$.

(ii) Assume that $\mathrm{H}^{1}(X, \mathbb{Q})$ is countable. If $\gamma$ is analytic, it is entirely determined by $\gamma(0)$ and by the curve $\mathrm{c}_{1}^{\mathrm{D}} \circ \gamma$ from $\Delta$ to $\mathrm{H}_{\mathrm{D}}^{2}(X, \mathbb{Q}(1))$, where $\mathrm{c}_{1}^{\mathrm{D}}$ is given by $(3.1)$.

Proof. (i) Since $\mathrm{H}^{1}\left(X, \mathcal{O}_{X}\right)$ is separated, we can apply the Künneth formula for Fréchet spaces (cf. [18] and [1, exposé 24]): we obtain that

$$
\mathrm{H}^{1}\left(X \times \Delta, \mathcal{O}_{X \times \Delta}\right)=\mathrm{H}^{1}\left(X, \mathcal{O}_{X}\right) \widehat{\otimes} \mathcal{O}(\Delta),
$$

where $\widehat{\otimes}$ denotes the topological tensor product. Besides, thanks to $[16$, Section 7.1 Proposition 6], the natural map from $\mathrm{H}^{1}\left(X, \mathcal{O}_{X}\right) \otimes \mathcal{O}(\Delta)$ to $\mathcal{O}\left\{\Delta, \mathrm{H}^{1}\left(X, \mathcal{O}_{X}\right)\right\}$ given by $\alpha \otimes f \rightarrow\{t \rightarrow f(t) \alpha\}$ extends to an isomorphism between $\mathrm{H}^{1}\left(X, \mathcal{O}_{X}\right) \widehat{\otimes} \mathcal{O}(\Delta)$ and $\mathcal{O}\left\{\Delta, \mathrm{H}^{1}\left(X, \mathcal{O}_{X}\right)\right\}$. Therefore, we get a natural isomorphism between $\mathrm{H}^{1}\left(X \times \Delta, \mathcal{O}_{X \times \Delta}\right)$ and $\mathcal{O}\left\{\Delta, \mathrm{H}^{1}\left(X, \mathcal{O}_{X}\right)\right\}$ obtained by attaching to each class $\beta$ in $\mathrm{H}^{1}\left(X \times \Delta, \mathcal{O}_{X \times \Delta}\right)$ the function $t \rightarrow \beta_{\mid X \times\{t\}}$. It is easy to conclude from there: if $\gamma-\gamma(0)$ can be lifted to an holomorphic curve with values in $\mathrm{H}^{1}\left(X, \mathcal{O}_{X}\right)$, then there exists a class $\beta$ in $\mathrm{H}^{1}\left(X \times \Delta, \mathcal{O}_{X \times \Delta}\right)$ such that for all $t$ in $\Delta$, the curve $t \rightarrow \beta_{\mid X \times\{t\}}$ lifts $\gamma-\gamma(0)$. Therefore the curve $\gamma$ comes from the class $\beta+\operatorname{pr}_{1}^{*} \gamma(0)$, so it is analytic. The converse implication is proven in the same way.

(ii) Assume that $\gamma$ is analytic, that $\gamma(0)=0$, and that for any $t$ in $\Delta$, the image of $\gamma(t)$ in $\mathrm{H}_{\mathrm{D}}^{2}(X, \mathbb{Q}(1))$ vanishes. If $\tilde{\gamma}$ is a holomorphic lift of $\gamma$ from $\Delta$ to $\mathrm{H}^{1}\left(X, \mathcal{O}_{X}\right)$ vanishing at the origin, then $\tilde{\gamma}(\Delta)$ lies in the image of $\mathrm{H}^{1}\left(X, \mathbb{Q}_{X}\right)$ in $\mathrm{H}^{1}\left(X, \mathcal{O}_{X}\right)$. Therefore $\tilde{\gamma}$ vanishes since it is continuous with values in a Hausdorff space and has countable image.

We can define the derivative of an analytic curve with values in $\operatorname{Pic}(X)$, it is a holomorphic curve with values in $\mathrm{H}^{1}\left(X, \mathcal{O}_{X}\right)$. Also not used elsewhere in the paper, the next proposition will provide another geometric interpretation of Proposition 3.1.

Proposition 3.5. Let $X$ be a complex manifold and assume that $\mathrm{H}^{1}\left(X, \mathcal{O}_{X}\right)$ is separated and that $\mathrm{H}^{1}(X, \mathbb{Q})$ is countable. Let $\gamma: \Delta \rightarrow \operatorname{Pic}(X)$ be an analytic curve given by a class $\alpha$ in $\operatorname{Pic}(X \times \Delta)$ and let $\hat{\gamma}$ be a holomorphic lift of $\gamma-\gamma(0)$ in $\mathrm{H}^{1}\left(X, \mathcal{O}_{X}\right)$. For any $t$, we define $\gamma^{\prime}(t)$ in $\mathrm{H}^{1}\left(X, \mathcal{O}_{X}\right)$ by putting $\gamma^{\prime}(t)=\hat{\gamma}^{\prime}(t)$. Then:

(i) The derivative of $\gamma$ is independant of $\hat{\gamma}$.

(ii) For any $t$ in $\Delta, \gamma^{\prime}(t)$ is the image of $\alpha$ by the composition

$\mathrm{H}_{\mathrm{D}}^{2}(X \times \Delta, \mathbb{Q}(1)) \longrightarrow \mathrm{H}^{1}\left(X \times \Delta, \Omega_{X \times \Delta}^{1}\right) \longrightarrow \mathrm{H}^{1}\left(X \times \Delta, \mathcal{O}_{X} \otimes \Omega_{\Delta}^{1}\right) \longrightarrow \mathrm{H}^{1}\left(X \times\{t\}, \mathcal{O}_{X}\right)$. 
(iii) If $\gamma^{\prime}$ vanishes identically then $\gamma$ is constant.

Proof. The argument of the proof of Proposition 3.4 (ii) shows that two holomorphic lifts of $\gamma$ differ by a constant. This gives the statement of point (i). Point (ii) results of the isomorphism between $\mathrm{H}^{1}\left(X \times \Delta, \mathcal{O}_{X \times \Delta}\right)$ and $\mathrm{H}^{1}\left(X, \mathcal{O}_{X}\right) \widehat{\otimes} \mathcal{O}(\Delta)$. For the point (iii), we use this last isomorphism to conclude that the vanishing of $\gamma^{\prime}$ implies that the image of the class $\alpha$ in $\mathrm{H}^{1}\left(X \times \Delta, \mathcal{O}_{X} \otimes \Omega_{\Delta}^{1}\right)$ is zero. Then we can apply Proposition 3.1 for integral Deligne cohomology.

\section{Proof of the main results}

4.1. Proof of Theorem 1.3. Let us consider the determinant line bundle $\lambda(\mathcal{E}, \hat{f})$ on $Y \times \Delta$, where $\hat{f}=f \times$ id. By Proposition 2.4, its restriction to a slice $Y \times\{t\}$ is $\lambda\left(\mathcal{E}_{t}, f\right)$. Therefore the curve $t \rightarrow \lambda\left(\mathcal{E}_{t}, f\right)$ is analytic. Let $\alpha$ be the class in $\mathrm{H}_{\mathrm{D}}^{2}(Y \times \Delta, \mathbb{Q}(1))$ defined by

$$
\alpha=\mathrm{c}_{1}^{\mathrm{D}}(\lambda(\mathcal{E}, \hat{f}))-\left(\hat{f}_{*}\left[\operatorname{ch}^{\mathrm{D}}(\mathcal{E}) \operatorname{td}^{\mathrm{D}}\left(\mathrm{T}_{X \times \Delta / Y \times \Delta}\right)\right]\right)^{(2)} .
$$

By Lemma 2.2,

$$
\mathrm{c}_{1}^{\mathrm{H}}(\lambda(\mathcal{E}, \hat{f}))=\sum_{p}(-1)^{p} \mathrm{c}_{1}^{\mathrm{H}}\left(\mathrm{R}^{p} \hat{f}_{*} \mathcal{E}\right)
$$

in $\mathrm{H}^{1}\left(Y \times \Delta, \Omega_{Y \times \Delta}^{1}\right)$. Therefore, thanks to the Grothendieck-Riemann-Roch theorem in Hodge cohomology [24], $\alpha$ maps to zero in $\mathrm{H}^{1}\left(Y \times \Delta, \Omega_{Y \times \Delta}^{1}\right)$. By Proposition 3.1, we obtain that for all $t$ in $\Delta, \alpha_{\mid Y \times\{t\}}$ does not depend on $t$.

It remains to compute $\alpha_{\mid Y \times\{t\}}$. If we denote by [.] the Deligne cohomology class of an analytic cycle, for any class $\beta$ in the rational Deligne cohomology ring of $X \times \Delta$, we have ${ }^{3}$

$$
\begin{aligned}
\left\{\hat{f}_{*} \beta\right\}_{\mid Y \times\{t\}} & =\operatorname{pr}_{1 *}\left\{\hat{f}_{*} \beta \cdot[Y \times\{t\}]_{\mathrm{D}}\right\}=\operatorname{pr}_{1 *} \hat{f}_{*}\left\{\beta \cdot \hat{f}^{*}[Y \times\{t\}]_{\mathrm{D}}\right\} \\
& =f_{*} \operatorname{pr}_{1 *}\left\{\beta \cdot[X \times\{t\}]_{\mathrm{D}}\right\}=f_{*}\left(\beta_{\mid X \times\{t\}}\right) .
\end{aligned}
$$

Then $\alpha_{\mid Y \times\{t\}}=\mathrm{c}_{1}^{\mathrm{D}}\left(\lambda\left(\mathcal{E}_{t}, f\right)\right)-\left(f_{*}\left\{\operatorname{ch}^{\mathrm{D}}\left(\mathcal{E}_{t}\right) \operatorname{td}^{\mathrm{D}}\left(\mathrm{T}_{X / Y}\right)\right\}\right)^{(2)}$. This gives (i). Remark now that for all $t$ in $\Delta$, we have

$$
\mathrm{c}_{1}^{\mathrm{D}}\left(\lambda\left(\mathcal{E}_{t}, f\right)\right)-\mathrm{c}_{1}^{\mathrm{D}}\left(\lambda\left(\mathcal{E}_{0}, f\right)\right)=\left(f_{*}\left\{\left[\operatorname{ch}^{\mathrm{D}}\left(\mathcal{E}_{t}\right)-\operatorname{ch}^{\mathrm{D}}\left(\mathcal{E}_{0}\right)\right] \operatorname{td}^{\mathrm{D}}\left(\mathrm{T}_{X / Y}\right)\right\}\right)^{(2)} .
$$

Thus, the curve $t \rightarrow\left(f_{*}\left(\left[\operatorname{ch}^{\mathrm{D}}\left(\mathcal{E}_{t}\right)-\operatorname{ch}^{\mathrm{D}}\left(\mathcal{E}_{0}\right)\right] \mathrm{td}^{\mathrm{D}}\left(\mathrm{T}_{X / Y}\right)\right)\right)^{(2)}$ in $\mathrm{H}_{\mathrm{D}}^{2}(Y, \mathbb{Q}(1))$ can be lifted to the analytic curve $\gamma: t \rightarrow \lambda\left(\mathcal{E}_{t}, f\right)-\lambda\left(\mathcal{E}_{0}, f\right)$ in $\operatorname{Pic}^{0}(Y)$. Thanks to Proposition 3.4 (ii), this lift is unique.

4.2. Proof of Theorem 1.4. Since $\mathcal{L}$ lies in $\operatorname{Pic}^{0}(Y \times F), \mathrm{c}_{1}^{\mathrm{D}}(\mathcal{L})^{i}=0$ for $i \geq 2$ so that

$$
p_{*}\left\{\mathrm{c}_{1}^{\mathrm{D}}(\mathcal{L}) q^{*} \operatorname{td}_{n}^{\mathrm{D}}(F)\right\}=\left(p_{*}\left\{\operatorname{ch}^{\mathrm{D}}(\mathcal{L}) \operatorname{td}^{\mathrm{D}}\left(\mathrm{T}_{Y \times F / Y}\right)\right\}\right)^{(2)} .
$$

Then we can conclude using Theorem 1.3. Indeed, since the line bundle $\mathcal{L}$ lies in $\operatorname{Pic}^{0}(Y \times F)$, there exists a holomorphic family of holomorphic line bundles joining

\footnotetext{
${ }^{3}$ This computation is a particular case of a base change type formula in Deligne cohomology, which is currently not available in the literature (in the analytic setting) for arbitrary base change morphisms.
} 
$\mathcal{O}_{Y \times F}$ to $\mathcal{L}$. Thanks to Theorem 1.3 , we are reduced to the case $\mathcal{L}=\mathcal{O}_{Y \times F}$. In this case, Theorem 1.4 is straightforward since

$$
\operatorname{det} \operatorname{R} p_{*}\left(\mathcal{O}_{Y \times F}\right) \simeq \operatorname{det}\left[\mathcal{O}_{Y} \stackrel{\mathrm{L}}{\otimes_{\mathbb{C}_{Y}}} \mathrm{R} \Gamma\left(F, \mathcal{O}_{F}\right)\right] \simeq \mathcal{O}_{Y}
$$

\section{Acknowledgments}

I would like to thank Andrei Teleman for suggesting the problem solved in this paper and for many comments, and also Christophe Soulé for kindly explaining to me some of the material of [6].

\section{References}

[1] Séminaire Schwartz de la Faculté des Sciences de Paris, 1953/1954. Produits tensoriels topologiques d'espaces vectoriels topologiques. Espaces vectoriels topologiques nucléaires. Applications, Secrétariat mathématique, 11 rue Pierre Curie, Paris, 1954.

[2] M.F. Atiyah and F. Hirzebruch, The Riemann-Roch theorem for analytic embeddings, Topology 1 (1962), 151-166.

[3] M.F. Atiyah and I.M. Singer, The index of elliptic operators, I, Ann. Math. 87(2) (1968), 484530.

[4] J.-M. Bismut, Hypoelliptic Laplacian and Bott-Chern cohomology, Springer, New York 2013.

[5] J.-M. Bismut and D.S. Freed, The analysis of elliptic families. II. Dirac operators, eta invariants, and the holonomy theorem, Comm. Math. Phys. 107(1) (1986), 103-163.

[6] J.-M. Bismut, H. Gillet and C. Soulé, Analytic torsion and holomorphic determinant bundles. III. Quillen metrics on holomorphic determinants, Comm. Math. Phys. 115(2) (1988), 301-351.

[7] J.-M. Bismut and K. Köhler, Higher analytic torsion forms for direct images and anomaly formulas, J. Algebr. Geom. 1(4) (1992), 647-684.

[8] A. Borel and J.-P. Serre, Le théorème de Riemann-Roch, Bull. Soc. Math. France 86 (1958) 97-136.

[9] P. Deligne, Le déterminant de la cohomologie, in 'Current trends in arithmetical algebraic geometry' (Arcata, CA, 1985), Vol. 67 of Contemp. Math., 93-177, Amer. Math. Soc., Providence, RI (1987).

[10] A. Dimca, Sheaves in topology, Universitext, Springer-Verlag, Berlin 2004, ISBN 3-540-20665-5.

[11] H. Esnault and E. Viehweg, Deligne-Beulinson cohomology, in 'Beǔlinson's conjectures on special values of L-functions', Vol. 4 of Perspect. Math., 43-91, Academic Press, Boston, MA (1988).

[12] S.I. Gelfand and Y.I. Manin, Homological algebra, Springer-Verlag, Berlin 1999, ISBN 3-54065378-3. Translated from the 1989 Russian original by the authors, Reprint of the original English edition from the series Encyclopaedia of Mathematical Sciences [1t Algebra, V, Encyclopaedia Math. Sci., 38, Springer-Verlag, Berlin, 1994; MR1309679 (95g:18007)].

[13] H. Grauert, Ein Theorem der analytischen Garbentheorie und die Modulräume komplexer Strukturen, Inst. Hautes Études Sci. Publ. Math. (5) (1960), 64.

[14] J. Grivaux, Chern classes in Deligne cohomology for coherent analytic sheaves, Math. Ann. $\mathbf{3 4 7}(2)$ (2010), 249-284.

[15] A. Grothendieck, Sur certains espaces de fonctions holomorphes, I, J. Reine Angew. Math. 192 (1953), 35-64.

[16] - Sur certains espaces de fonctions holomorphes, II, J. Reine Angew. Math. 192 (1953), $77-95$.

[17] M. Kashiwara and P. Schapira, Deformation quantization modules, Astérisque, (345) (2012), xii +147 .

[18] L. Kaup, Eine Künnethformel für Fréchetgarben, Math. Z. 97 (1967), 158-168.

[19] F.F. Knudsen and D. Mumford, The projectivity of the moduli space of stable curves. I. Preliminaries on "det" and "Div", Math. Scand. 39(1) (1976), 19-55. 
[20] S. Kobayashi, Differential geometry of complex vector bundles, Vol. 15 of Publications of the Mathematical Society of Japan, Princeton University Press, Princeton, NJ (1987), ISBN 0-69108467-X. Kanô Memorial Lectures, 5.

[21] C. Laurent-Thiébaut and J. Leiterer, A separation theorem and Serre duality for the Dolbeault cohomology, Ark. Mat. 40(2) (2002), 301-321.

[22] R.N. Levy, The Riemann-Roch theorem for complex spaces, Acta Math. 158(3-4) (1987), $149-188$.

[23] N.R. O'Brian, D. Toledo, and Y.L.L. Tong, Hirzebruch-Riemann-Roch for coherent sheaves, Amer. J. Math. 103(2) (1981), 253-271.

[24] —, A Grothendieck-Riemann-Roch formula for maps of complex manifolds, Math. Ann. 271(4) (1985), 493-526.

[25] V.K. Patodi, An analytic proof of Riemann-Roch-Hirzebruch theorem for Kaehler manifolds, J. Differ. Geom. 5 (1971), 251-283.

[26] D. Quillen, Determinants of Cauchy-Riemann operators on Riemann surfaces, Funktsional. Anal. Prilozhen. 19(1) (1985), 37-41, 96.

[27] H. Rossi, Attaching analytic spaces to an analytic space along a pseudoconcave boundary, in 'Proc. Conf. Complex Analysis' (Minneapolis, MN, 1964), 242-256, Springer-Verlag, Berlin 1965.

[28] J.-P. Serre, Faisceaux algébriques cohérents, Ann. Math. 61(2) (1955), 197-278.

[29] — Géométrie algébrique et géométrie analytique, Ann. Inst. Fourier, Grenoble 6 (1955-1956), 1-42.

[30] C. Soulé, Lectures on Arakelov geometry, Vol. 33 of Cambridge Studies in Advanced Mathematics, Cambridge University Press, Cambridge (1992), ISBN 0-521-41669-8. With the collaboration of D. Abramovich, J.-F. Burnol and J. Kramer.

[31] A. Teleman, Donaldson theory on non-Kählerian surfaces and class VII surfaces with $b_{2}=1$, Invent. Math. 162(3) (2005), 493-521.

[32] - Instantons and curves on class VII surfaces, Ann. Math. (2) 172(3) (2010), 1749-1804.

[33] D. Toledo and Y.L.L. Tong, A parametrix for $\bar{\partial}$ and Riemann-Roch in Čech theory, Topology 15(4) (1976), 273-301.

[34] C. Voisin, Hodge Theory and Complex Algebraic Geometry, I, Vol. 76 of Cambridge Studies in Advanced Mathematics, Cambridge University Press, Cambridge, english edition, 2007, ISBN 978-0-521-71801-1. Translated from the French by Leila Schneps.

CNRS \& I2M (UMR 7373), CMI, Université D'AiX-Marseille, 39 Rue Frédéric Joliot-

Curie, 13453 Marseille Cedex 13, France

E-mail address: jgrivaux@math.cnrs.fr 
